\title{
Density response to magnetic field fluctuation in the foreshock plasma
}

\author{
Yasuhito Narita ${ }^{1 *}$ (D) and Tohru Hada 2,3
}

\begin{abstract}
The foreshock plasma exhibits large-amplitude disturbance in the plasma density and the magnetic field. The question of the density response to the magnetic field fluctuation is addressed and studied observationally and statistically using the in situ Cluster spacecraft data of the foreshock plasma. Three major findings are obtained. First, the density response is unique to the magnetic field fluctuation and is of the fast magnetosonic mode type. Second, the density response to the total magnetic energy density (simply subtracting by the mean field) exhibits a clear scaling to the beta-tilde parameter defined as the squared ratio of the sound speed to the Alfvén speed. We interpret that the total fluctuations mostly represent linear-mode waves, and the scaling law has a potential application to estimate the plasma parameter beta using the fluctuation data of the density and the magnetic field only. Third, the density response to the nonlinear (or large-amplitude) magnetic field fluctuation has a weak agreement with the theoretical expectation from the quasi-static balance with a larger degree of scattering in the data. We conclude that the foreshock plasma overall exhibits the linear-mode waves (fast mode) and a moderate degree of nonlinear fluctuations. The concept of the quasi-static balance is partly justified and applicable in the foreshock plasma.
\end{abstract}

Keywords: Foreshock plasma, Density fluctuation

\section{Origin of density fluctuations in space plasma}

Density fluctuations appear in various space plasma environments and are often accompanied by magnetic field fluctuations. Naively speaking, the sense of the density fluctuation can be correlated to that of the magnetic field fluctuation like in the fast magnetosonic mode or anticorrelated like in the slow mode or in the mirror mode. Various mechanisms are possible to cause the density fluctuations in association with the magnetic field, e.g., linear mode waves (magnetohydrodynamic waves), kinetic instabilities (mirror mode under temperature anisotropy), beating or modulation of the wave, ponderomotive force (pinching of the plasma by large-amplitude fluctuation), and shock and shocklet formation.

Above all, the hypothesis of quasi-static balance or quasi-static approximation offers a unified view of the density fluctuation such that fast magnetosonic mode

\footnotetext{
*Correspondence: yasuhito.narita@oeaw.ac.at

${ }^{1}$ Space Research Institute, Austrian Academy of Sciences, Schmiedlstraße 6, 8042 Graz, Austria

Full list of author information is available at the end of the article
}

(or a positive) correlation sense is realized in a lowbeta plasma, and the slow mode (or a negative) correlation sense in a high-beta plasma through the following relation:

$$
\frac{\delta n}{n_{0}}=\frac{1}{2\left(1-c_{\mathrm{s}}^{2} / V_{\mathrm{A}}^{2}\right)} \frac{\delta B_{\mathrm{nl}}^{2}}{B_{0}^{2}},
$$

which was first introduced by Cohen and Kulsrud (1974) and later revisited by Hada (1993), where $\delta n$ denotes the number density fluctuation, $n_{0}$ the mean density, $\delta B_{\mathrm{nl}}^{2}$ the nonlinear fluctuation of the squared magnetic field magnitude (which is a measure of the magnetic energy density), $B_{0}$ the mean magnetic field magnitude, $c_{\mathrm{S}}$ the sound speed, and $V_{\mathrm{A}}$ the Alfvén speed.

The notion of the quasi-static balance is a useful tool and an important subject in the theory of derivative nonlinear Schrödinger equation (DNLS) describing largeamplitude parallel-propagating Alfvén waves to the mean magnetic field (Mjølhus 1974, 1976; Spangler 1986, 1990) and phase coherence in Alfvén wave turbulence 
(Nariyuki and Hada 2005). DNLS is also extended to including kinetic effects (Medvedev and Diamond 1996; Medvedev et al. 1997).

Here we present for the first time a systematic and statistic observational study of the density response to the magnetic field fluctuation in the foreshock plasma. The study is performed in the spirit of testing for the quasi-static balance against in situ plasma and magnetic field data in nearEarth space. The foreshock plasma is a turbulent plasma upstream of the Earth (or planetary) bow shock. The foreshock plasma is connected to the bow shock by the magnetic field lines such that the shock-reflected ions stream backward against the solar wind flow and excite low frequency waves through the right-hand resonant ion beam instability or component/component instability (Gary 1991, 1993). Even though the driving mechanism of the waves is a kinetic process (beam instability associated with an unstable distribution function in the particle velocity space), the excited waves have sufficiently low frequencies in the frame of the thermal or core component of the plasma, lower than the ion gyro-frequency.

The foreshock waves are typically observed at a period of about $30 \mathrm{~s}$ in the spacecraft frame of reference (Greenstadt et al. 1995). The foreshock region serves as a natural laboratory for studying the nonlinear behavior of largeamplitude parallel-propagating waves. The earlier study by Stasiewicz et al. (2003) revealed the association of the density fluctuation with the magnetic field fluctuation in the bow shock or the shocklet structure, to be more exactly (Stasiewicz et al. 2003). To our knowledge, on the other hand, there is no earlier study which directly tackles on the relation between the density variation and that of the magnetic energy in the foreshock region in view of quasi-static approximation. Technically speaking, it is novel to study the correlation sense for the two fluctuating fields in association with the beta dependence systematically and statistically.

\section{Data set and analysis method}

\section{Cluster spacecraft in the foreshock}

The fluctuation sense is studied using the ion number density data and the magnetic field data from two Cluster spacecraft, Cluster-1 and Cluster-3, in the Earth foreshock region for a time period from 2 February 2002 to 31 May 2002. Two Cluster spacecraft are used for an enhanced statistical confidence. Cluster spacecraft (Escoubet et al. 2001) has an apogee of about 20 Earth radii ahead of the Earth (and ahead of the Earth bow shock). The Cluster orbits are ideal to study the foreshock plasma. The ion number density is measured by Cluster Ion Spectrometry instrument (CIS) (Rème et al. 2001) at a time resolution of spacecraft spin (about $4 \mathrm{~s}$ ). The magnetic field is measured by Fluxgate Magnetometer (FGM)
(Balogh et al. 2001) at a time resolution of about $22 \mathrm{~Hz}$. For the sake of the fluctuation sense analysis, the both data are time-synchronized to a 4-s resolution either by interpolation (for the density data) or by down-sampling through averaging (for the magnetic field data). We use the single spacecraft method. Two spacecraft data are used to improve the degree of freedom or the ensemble size in the statistical study. The averaging is made over the time domain on the time averaging is representative of the statistical averaging. Plasma beta parameter is determined by using the parallel and perpendicular ion temperatures obtained from the CIS experiment. The data for the electrons are not available on spacecraft $\mathrm{C}-1$ and $\mathrm{C}-3$. We assume the quasi-neutrality of plasma holds well with the time resolution of $4 \mathrm{~s}$. Estimate of the beta parameter is restricted to the ion measurements. Variation in (unmeasured) electron temperature may introduce a change in the beta parameter that is relevant to the test for the quasi-static approximation.

Cluster orbit period is about $60 \mathrm{~h}$, corresponding to 10 encounters of the foreshock region per month. The foreshock region is identified in the Cluster data by imposing the following conditions: (1) existence of the back-streaming ions in the ion energy spectra (using CIS data), (2) large-amplitude disturbance (using FGM data), (3) foreshock interval bounded by the bow shock crossing and the solar wind period. A sufficiently long time interval is split into a multitude of 40-min subintervals with a time separation of $2 \mathrm{~h}$ from one to another. The event list is a refinement of that used in the earlier foreshock wave studies (Narita et al. 2004, 2007). The number of data points on each interval is set to 600 (equivalent to 40-min data length), with which the standard error estimate in the statistical analysis can be suppressed down to about $4 \%$ of the mean value estimate (using a scaling of the standard error, $N^{-1 / 2}$, where $N$ denotes the degree of freedom in ensemble averaging). After the data survey, 25 foreshock intervals with a time length of 40 min are identified and registered into the foreshock event list.

\section{Fluctuation sense analysis}

The data analysis procedure takes the following steps.

1. Time series data for the ion number density $n_{\mathrm{i}}$ and the squared magnetic field magnitude $B^{2}$ are transformed into the relative fluctuation amplitude to the mean field as $\delta n_{\mathrm{i}} / n_{\mathrm{i} 0}=\left(n_{\mathrm{i}}-n_{\mathrm{i} 0}\right) / n_{\mathrm{i} 0}$ for the density data and $\delta B_{\mathrm{t}}^{2} / B_{0}^{2}=\left(B^{2}-B_{0}^{2}\right) / B_{0}^{2}$ for the magnetic field data. The subscript " $\mathrm{t}$ " denotes the total fluctuation field. Hereafter, the fluctuation is referred to as the total fluctuation in the sense that both linear-mode and nonlinear fluctuations are included in the data. 
2. Nonlinear fluctuation data for the magnetic field (or the magnetic energy density, strictly speaking) are derived from the total fluctuation data by subtracting by the variance as $\delta B_{\mathrm{nl}}^{2} / B_{0}^{2}=\left(\delta B_{\mathrm{t}}^{2}-\left\langle\delta B^{2}\right\rangle\right) / B_{0}^{2}$, following the treatment introduced by Cohen and Kulsrud (1974). Here the angular bracket denotes the averaging. It is worth mentioning that the sense of density fluctuation $\delta n_{\mathrm{i}}$ is compared to the magnetic energy density $\delta B^{2}$ in our work, and not the linear field $\delta B$, because the pressure variation is proportional to the number density such that $\nabla p=c_{\mathrm{s}}^{2} \nabla \rho$ on the assumption of the adiabatic condition, $p / \rho^{\gamma}=$ const. (where $p$ is the pressure, $\rho$ the mass density, and $\gamma$ the polytropic index), and the magnetic energy density is proportional to the squared field magnitude. The mean fields $\left(n_{\mathrm{i} 0}\right.$ for the density and $B_{0}$ for the magnetic field) are obtained by averaging the data over the time domain (assumed to be constant over time). Both the total fluctuation data $\delta B_{\mathrm{t}}^{2} / B_{0}^{2}$ and the nonlinear fluctuation data $\delta B_{\mathrm{nl}}^{2} / B_{0}^{2}$ are used to study the density response to the magnetic field variation.

3. The density response to the magnetic field fluctuation is graphically and qualitatively studied by plotting the density fluctuation data as a function of the total magnetic field fluctuation in a scatter plot diagram. Examples are displayed in Figs. 1 and 2.

4. The density response to the magnetic field fluctuation is quantitatively studied by determining the slope or the coefficient of the linear relation between the density fluctuation $\delta n$ and the total magnetic field fluctuation $\delta B_{\mathrm{t}}^{2}$ by using the least square fitting to a linear relation, minimizing the Chi-square quantity (Press et al. 1992). Both the total fluctuation data $\delta B_{\mathrm{t}}^{2}$ and the nonlinear fluctuation data $\delta B_{\mathrm{nl}}^{2}$ are used.

5. The values of the slope are associated with the plasma parameter beta for each event. Here, we introduce the beta-tilde parameter defined as the squared ratio of the sound speed $c_{\mathrm{s}}$ to the Alfvén speed $V_{\mathrm{A}}$ such that $\tilde{\beta}=c_{\mathrm{s}}^{2} / V_{\mathrm{A}}^{2}$. The squared sound speed is computed using the relation $c_{\mathrm{s}}^{2}=\gamma k_{\mathrm{B}} T / m_{\mathrm{i}}$, where $\gamma=5 / 3$ is the polytropic index, $m_{\mathrm{i}}$ the ion mass (for protons), $k_{\mathrm{B}}$ the Boltzmann constant, and $T=\left(T_{\|}+2 T_{\perp}\right) / 3$ the scalar temperature for ions. The temperatures parallel and perpendicular to the mean magnetic field direction are obtained by the CIS experiment on board Cluster- 1 and Cluster- 3 . Note that the electron data are not available in the data set we use here. The squared Alfvén speed is computed through the relation $V_{\mathrm{A}}^{2}=B_{0}^{2} /\left(\mu_{0} m_{\mathrm{i}} n_{0}\right)$, where $\mu_{0}$ is the permeability of free space. The beta-tilde is related to the plasma parameter beta (the ratio of the thermal pressure to the magnetic pressure) by $\tilde{\beta} / \beta=\sqrt{\gamma / 2}$.

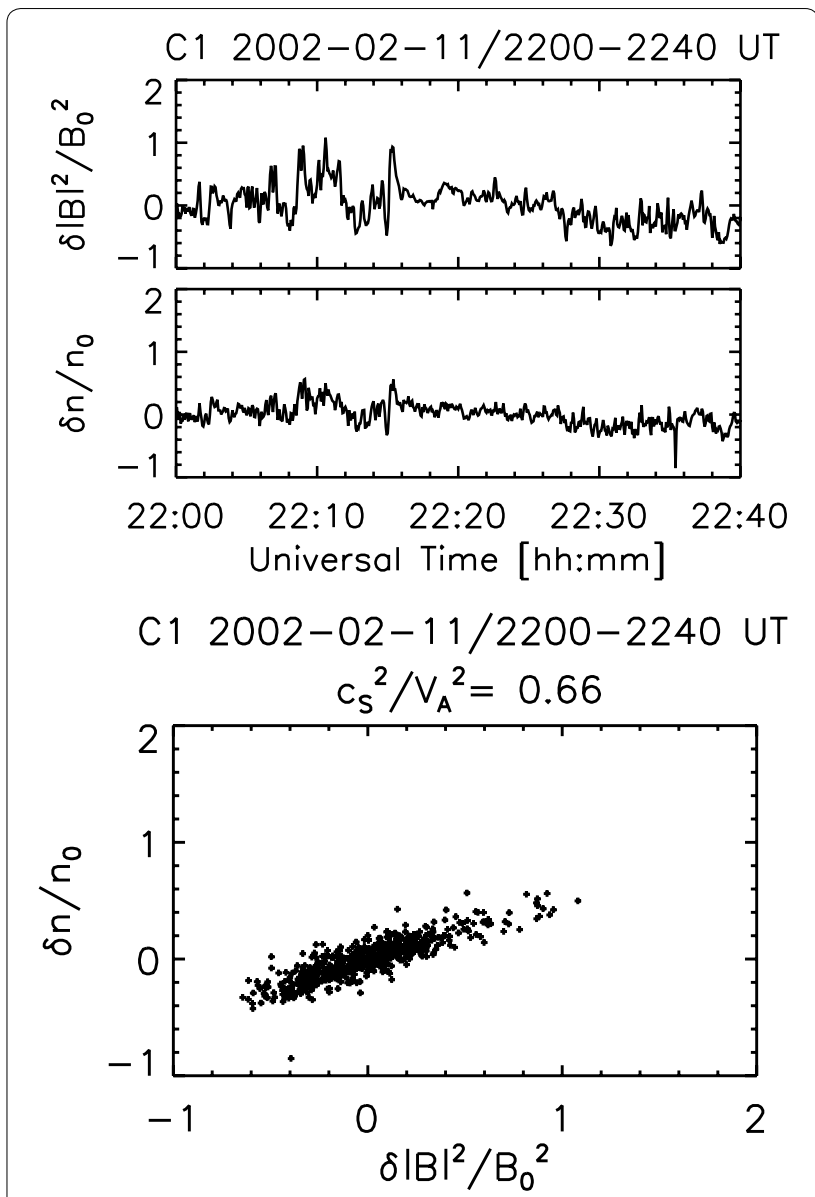

Fig. 1 Time series plot of the fluctuations around the mean field for the magnetic energy density $\delta\left|B^{2}\right|$ (top panel) and the ion number density $\delta n_{\mathrm{i}}$ (second panel) measured by Cluster-1 spacecraft on 11 February 2002, 2200-2240 UT, and the scatter plot of the two fields (third panel). Value of the ion beta is 0.66

6. The slope diagram is constructed by plotting the slope of the density-magnetic field relation from the linear fitting (procedure 4 ) as a function of the betatilde parameter for the total fluctuation $\delta B_{\mathrm{t}}^{2}$ (Fig. 3) and the nonlinear fluctuation $\delta B_{\mathrm{nl}}^{2}$ (Fig. 4).

\section{Results}

\section{Sense of density response}

Qualitative nature of the density response to the magnetic field fluctuation is in a positive correlation sense like fluctuations in the fast magnetosonic mode. The positive correlation sense is observed both in the low-beta and high-beta foreshock plasmas. Examples are displayed in Fig. 1 for a low-beta plasma and in Fig. 2 for a high-beta plasma. Time series plots of the relative magnetic energy 

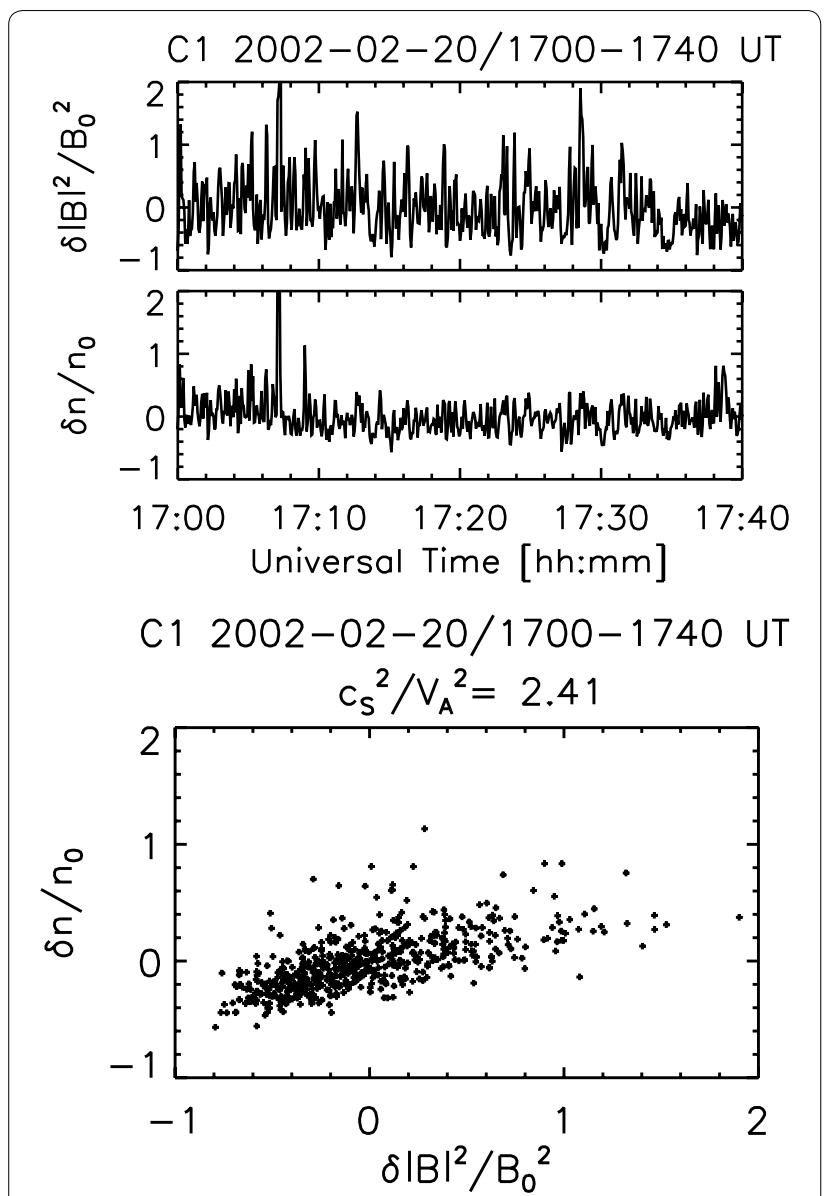

Fig. 2 The same panel style as Fig. 1 for a high-beta foreshock event on 20 February, 2002, 1700-1740 UT. Value of the ion beta is 2.41

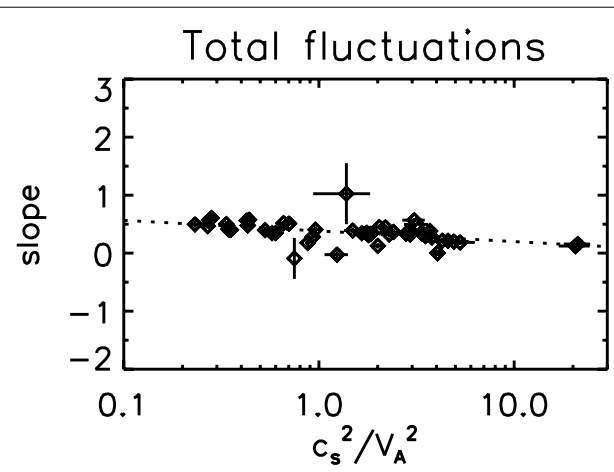

Fig. 3 Slope of the linear relation between the density fluctuation and the squared magnetic field fluctuation plotted as a function of the beta-tilde parameter $\left(\tilde{\beta}=c_{\mathrm{s}}^{2} / V_{\mathrm{A}}^{2}\right)$. The slope is evaluated using the density fluctuation and the total magnetic field fluctuation (including both linear fluctuations and nonlinear fluctuations) by subtracting by the mean magnetic field, $\delta B_{t}^{2}=B^{2}-B_{0}^{2}$. The slope values are derived from the least square fitting: single response group in black, separate response group in diamond and gray, and overlapped response group in triangle and gray. Error bars indicated three-sigma for a confidence interval of about $95 \%$ both in the beta-tilde parameter and the slope estimates fluctuation $\delta B_{\mathrm{t}}^{2} / B_{0}^{2}$ and that of the ion number density $\delta n_{\mathrm{i}} / n_{\mathrm{i} 0}$ are displayed in the first and second panels.

The correlation sense is studied by plotting the fluctuation amplitudes in a scatter plot diagram for the magnetic energy and the ion number density. The mapping is nearly unique between the ion number density fluctuation and that of the magnetic energy density within the statistical uncertainty. The fitting to a linear relation is applied to the density-magnetic field data,

$$
\frac{\delta n_{\mathrm{i}}}{n_{\mathrm{i} 0}}=c_{1} \frac{\delta B_{\mathrm{t}}^{2}}{B_{0}^{2}}+c_{0}
$$

where $c_{0}$ is the offset or the ordinate of the relation and $c_{1}$ the slope coefficient. The fitting is performed by minimizing Chi-square error.

We obtain positive values of the slope coefficient, $0.518 \pm 0.037$ in the both low-beta case (Fig. 1) and $0.367 \pm 0.019$ in the high-beta case (Fig. 2). The error bar here represents a three-sigma value (for a nearly $95 \%$ confidence). A higher level of data scattering in the highbeta case (Fig. 2) than that in the low-beta case (Fig. 1) may as well originate in the co-existence of different wave modes or different stages of nonlinear wave evolution.

\section{Beta dependence}

The slope values of the fluctuation sense between the ion number density and the magnetic energy density are plotted against the beta-tilde parameter for the total fluctuations of the magnetic field (Fig. 3) and the nonlinear fluctuation of the magnetic field (Fig. 4).

In the slope diagram for the total fluctuations (Fig. 3), the slope values are overall positive and exhibit the character of fast magnetosonic mode irrespective of the value of the beta-tilde parameter in a range of $0.1<\tilde{\beta}<22$. Only few events exhibit barely negative slope values. There is a systematic trend in the diagram for the total fluctuations that the slope of the linear relation is steeper at lower values of $\tilde{\beta}$ (below unity) and flatter at higher values of $\tilde{\beta}$ (above unity). The slope can virtually uniquely be mapped to a given value of $\tilde{\beta}$.

An empirical relation between the density and the magnetic field can indeed be obtained for the total fluctuations as $\delta n / n_{0} \propto c_{1} \delta B^{2} / B_{0}^{2}$, and the slope $c_{0}$ is associated with the beta-tilde parameter through $c_{1}=a_{1} \log _{10}\left(c_{\mathrm{s}}^{2} / V_{\mathrm{A}}^{2}\right)+a_{0}$ with the coefficients $a_{1}=-0.183 \pm 0.153$ (which is the empirical scaling of the slope value to the beta-tilde) and $a_{0}=0.383 \pm 0.076$ (the offset of the slope value). Here error bars represent again three-sigma values.

In the slope diagram for the nonlinear fluctuations (Fig. 4), the slope values have a larger scattering over the 


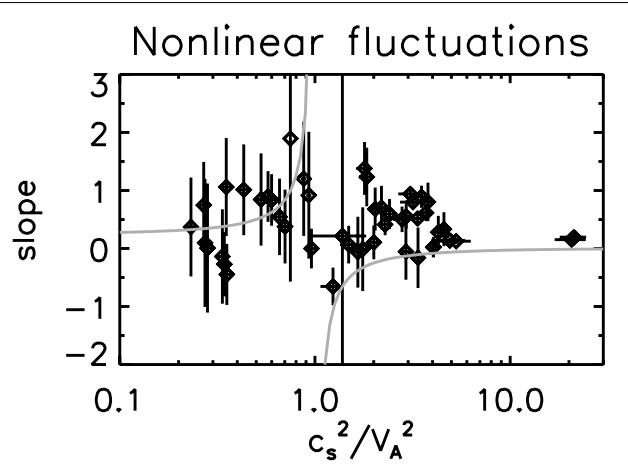

Fig. 4 The same panel as Fig. 3 but the slope is evaluated for the second-order nonlinear magnetic field fluctuation by subtracting by variance of the magnetic field fluctuation, $\delta B_{\mathrm{nl}}^{2}=\delta B_{\mathrm{t}}^{2}-\left\langle\delta B_{\mathrm{t}}^{2}\right\rangle$. Curved lines in gray are the prediction from the quasi-static balance with a slope relation to the beta-tilde $(2(1-\tilde{\beta}))^{-1}$

beta-tilde domain. Error bars are larger than that for the total fluctuation, too. The slope diagram is compared to the relation between the density fluctuation and the nonlinear magnetic field fluctuation derived from the theoretical expectation for the quasi-static balance (Cohen and Kulsrud 1974), $\delta n / n_{0}=(2(1-\tilde{\beta}))^{-1} \delta B_{\mathrm{nl}}^{2} / B_{0}^{2}$. Some data points agree in the low beta values $(\tilde{\beta}<0.5)$, but the divergent shape of the slope values around $c_{\mathrm{s}}^{2} / V_{\mathrm{A}}^{2}=1$ is not confirmed in the measurement.

We interpret that the concept of the quasi-static approximation is partly applicable in the foreshock plasma. Needless to say, an extended work with a larger number of foreshock plasma samples is ideal to validate our discoveries (empirical scaling for the total fluctuations; partial agreement with the quasi-static approximation).

Various reasons are possible to explain the mismatch from the theoretical curve for the quasi-static approximation. First of all, our identification of the nonlinear fluctuations follows the definition by Cohen and Kulsrud (1974). One needs to subtract the linear-mode fluctuations and the wave mode identification is necessary to more clearly extract the nonlinear fluctuations from the measured fluctuations. Second, the concept of the quasi-static approximation originates in the fluid treatment of the foreshock plasma. Kinetic treatment by including particle motions and velocity distribution functions is certainly more suited to describe the foreshock plasma. Third, the ion number density data are used in our analysis. Electrons also contribute to the plasma temperature, and need to be included in further studies.

\section{Lessons}

Major findings or lessons about the density response to the magnetic field fluctuation in the foreshock plasma are summarized with discussions and hints for further tests or applications as follows.
1. The sense of the density fluctuation is positively correlated to that of the magnetic energy (squared magnetic field magnitude) both in the low-beta and highbeta plasmas, reminiscent of the fast magnetosonic mode.

2. The slope diagram for the total magnetic field fluctuation has a well-defined, clear scaling to the betatilde parameter (the ratio of the sound speed to the Alfvén speed). An empirical scaling law is established from the least square fitting to the measurement data. The total fluctuations contain a large number of linear-mode waves. We interpret that the clear relationship in the first-order fluctuations between the density and the magnet energy density represents a property of the linear instability mechanism. Gary (1991) reviews the ion beam instabilities under different conditions such as the beam temperature and the core temperature anisotropy, while keeping the core beta value as unity (Gary 1991). Beta dependence of the fluctuation sense in the ion instability is an interesting discovery in our study and will be a subject for the linear instability analysis, e.g., using a dispersion solver.

3. An extrapolation of our result in Fig. 3 indicates that anti-correlation may be realized between the density fluctuation and that of the magnetic energy. On the other hand, there is only one time interval at a beta value above 10 in our study, we cannot make clear prediction if the empirical relation can safely be extrapolated to such high beta plasmas. An extension of the statistical volume in the data analysis or the linear instability analysis would be necessary to verify the high-beta limit of our empirical relation.

4. It would be an interesting task to study theoretically the beta dependence of the ion beam instability, in particular, if one can explain or reproduce the empirical scaling from plasma kinetic theories. Kinetic theory predicts that the pressure needs to be treated as a tensor, that is, not only the pressure but also the shear stress (e.g., bending and torsion) is important in the nonlinear behavior (Spangler 1990). In contrast, the concept of pressure is often used as a scalar quantity in the MHD picture.

5. The finding of the scaling law for the density response to the total magnetic field fluctuation has an immediate, interesting application to spacecraft measurements, that is, the value of beta can be estimated solely from the fluctuation data for the density and the magnetic field. If the density response to the magnetic field fluctuation is well understood and calibrated, one may use only the fluctuating fields to determine or constrain the beta value of the foreshock plasma using the scaling law. In other words, 
one may be able to constrain the mean values such as the magnetometer offset value in the measurements.

6. The slope diagram for the nonlinear magnetic fluctuation does not exhibit a clear organization in the beta-tilde domain, at least in the data set we used. Agreement with the quasi-static balance is poor except for the low-beta values. Various reasons are possible to explain the different result from the expected curve from the quasi-static balance. For example, not waves but shocklets are being formed in the foreshock and the correlation sense remains positive irrespective of the value of beta, or the notion of the quasi-static balance needs to be extended to include kinetic or other effects such as temperature gradients or Landau damping. Magnetosonic-type low-frequency waves in the foreshock region are typically observed or recognized as $30-\mathrm{s}$ waves, $1-\mathrm{Hz}$ waves, shocklets, and SLAMS (short large-amplitude magnetic structures) (Greenstadt et al. 1995). It is considered that the initially transverse 30-s waves with upstream propagation in the plasma frame become compressive under a refraction process. The compressive fluctuation results into steepening and low frequency steepened magnetosonic whistler modes can be formed (the so-called shocklets).

7. A slight phase shift is reported between the magnetic field fluctuation and the density fluctuation using the Cluster measurements (Stasiewicz et al. 2003), in which the nonlinear momentum conservation equation of plasma fluid dynamics plays an important role. The magnetic energy is expressed as a function of the density, the polytropic index, the Mach number, and the beta parameter in the Stasiewicz model. Evaluation of the Stasiewicz model and investigation of the phase shift (between the magnetic field fluctuation and the density fluctuation) are an interesting logical extension of our study and will reveal the foreshock fluctuation property in more detail. The coefficient $c_{0}$ and $c_{1}$ may be extended to complex numbers, as well.

\section{Authors' contributions}

YN was involved in data analysis and writing. TH contributed to original idea, guiding the research, and interpretation of the results. All authors read and approved the final manuscript.

\footnotetext{
Author details

1 Space Research Institute, Austrian Academy of Sciences, Schmiedlstraße 6, 8042 Graz, Austria. ${ }^{2}$ Interdisciplinary Graduate School of Engineering Sciences, Kyushu University, Kasuga-koen 6-1, Kasuga City, Fukuoka Prefecture 816-8580, Japan. ${ }^{3}$ International Center for Space Weather Science and Education, Kyushu University, Motooka, Nishi-ku, Fukuoka City, Fukuoka Prefecture 819-0395, Japan.
}

\section{Acknowledgments}

YN acknowledges the hospitality of TH's research group at Kyushu University during the research visit in December 2017.

\section{Competing interests}

The authors declare that they have no competing interests.

\section{Availability of data and materials}

Cluster spacecraft data are available at Cluster Science Archive (URL at https:// csa.esac.esa.int/).

\section{Funding}

YN'work is financially supported by Invitational Fellowship for Research in Japan (short-term) under FY2017-S17123.

\section{Publisher's Note}

Springer Nature remains neutral with regard to jurisdictional claims in published maps and institutional affiliations.

Received: 25 July 2018 Accepted: 22 October 2018

Published online: 29 October 2018

\section{References}

Balogh A et al (2001) The Cluster magnetic field investigation: overview of in-flight performance and initial results. Ann Geophys 19:1207-1217. https://doi.org/10.5194/angeo-19-1207-2001

Cohen RH, Kulsrud RM (1974) Nonlinear evolution of parallel-propagating hydromagnetic waves. Phys Fluids 17:2215-2225. https://doi. org/10.1063/1.1694695

Escoubet CP, Fehringer M, Goldstein M (2001) The Cluster mission. Ann Geophys 19:1197-1200. https://doi.org/10.5194/angeo-19-1197-2001

Gary SP (1991) Electromagnetic ion/ion instabilities and their consequences in space plasmas: a review. Space Sci Rev 56:373-415. https://doi. org/10.1007/BF00196632

Gary SP (1993) Theory of space plasma microinstabilities. Cambridge University Press, Cambridge

Greenstadt EW, Le G, Strangeway RJ (1995) ULF waves in the foreshock. Adv Space Res 15:71-84. https://doi.org/10.1016/0273-1177(94)00087-H

Hada T (1993) Evolution of large amplitude Alfvén waves in the solar wind with $\beta \sim 1$. Geophys Res Lett 20:2415-2418. https://doi. org/10.1029/93GL02704

Medvedev MD, Diamond PH (1996) Fluid models for kinetic effects on coherent nonlinear Alfvén waves. Phys Plasmas 3:863-873. https://doi. org/10.1063/1.871790

Medvedev MD, Diamond PH, Shevchenko VI, Galinsky VL (1997) Dissipative dynamics of collisionless nonlinear Alfvén wave trains. Phys Rev Lett 78:4934-4937. https://doi.org/10.1103/PhysRevLett.78.4934

Mjølhus E (1974) Application of the reductive perturbation method to long hydromagnetic waves parallel to the magnetic field in a cold plasma, Report no. 48. Department of Applied Mathematics, University Bergen, Norway

Mjølhus E (1976) On the modulational instability of hydromagnetic waves parallel to the magnetic field. J Plasma Phys 16:321-334. https://doi. org/10.1017/S0022377800020249

Narita Y et al (2004) Alfvén waves in the foreshock propagating upstream in the plasma rest frame: statistics from Cluster observations. Ann Geophys 22:2315-2323. https://doi.org/10.5194/angeo-22-2315-2004

Narita Y, Glassmeier K-H, Fränz M, Nariyuki Y, Hada T (2007) Observations of linear and nonlinear processes in the foreshock wave evolution. Nonlinear Process Geophys 14(361-371):2007. https://doi.org/10.5194/ npg-14-361-2007

Nariyuki Y, Hada T (2005) Self-generation of phase coherence in parallel Alfvén turbulence. Earth Planets Space 57:e9-e12. https://doi.org/10.1186/ BF03352580

Press WH, Teukolsky SA, Vetterling SA, Flannery BP (1992) Numerical recipes in C, the art of scientific computing, sec. 15.2, 2nd edn. Cambridge University Press, Cambridge 
Rème $\mathrm{H}$ et al (2001) First multispacecraft ion measurements in and near the Earth's magnetosphere with the identical Cluster ion spectrometry (CIS) experiment. Ann Geophys 19:1303-1354. https://doi.org/10.5194/angeo -19-1303-2001

Spangler SR (1986) The evolution of nonlinear Alfvén waves subject to growth and damping. Phys Fluids 29:2535-2547. https://doi.org/10.1063/1.86554 5
Spangler SR (1990) Kinetic effects on Alfvén-wave nonlinearity. II. The modified nonlinear-wave equation. Phys Fluids B 2:407-418. https://doi. org/10.1063/1.859329

Stasiewicz K, Longmore M, Buchert S, Shukla PK, Lavraud B, Pickett J (2003) Properties of fast magnetosonic shocklets at the bow shock. Geophys Res Lett 30:2241. https://doi.org/10.1029/2003GL017971

\section{Submit your manuscript to a SpringerOpen ${ }^{\circ}$ journal and benefit from:}

- Convenient online submission

- Rigorous peer review

- Open access: articles freely available online

- High visibility within the field

- Retaining the copyright to your article

Submit your next manuscript at $\boldsymbol{\nabla}$ springeropen.com 